\title{
Reconsidering the mechanistic basis of metabolic theory of ecology
}

\author{
Article in Oikos · June 2007
}

DOI: 10.1111/j.2006.0030-1299.15534.x

CITATIONS

64

8 authors, including:

\section{Stanley J. Kemp}

University of Baltimore

13 PUBLICATIONS 358 CITATIONS

SEE PROFILE

\section{James N. McNair}

Grand Valley State University

40 PUBLICATIONS $\mathbf{1 , 1 8 7}$ CITATIONS

$$
\text { SEE PROFILE }
$$

READS

149

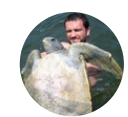

Bryan P Wallace

Conservation Science Partners, Inc.

84 PUBLICATIONS 2,396 CITATIONS

SEE PROFILE

\section{Arthur E Dunham}

University of Pennsylvania

80 PUBLICATIONS 5,571 CITATIONS

SEE PROFILE

Some of the authors of this publication are also working on these related projects:

Project

The Stockholm Paradigm: testing the elements of a paradigm in evolution using theoretical and empirical data View project

All content following this page was uploaded by Bryan P Wallace on 16 June 2017. 
Oikos 000: 000-000, 2007

doi: 10.1111/j.2006.0030-1299.15534.x Copyright (C) Oikos 2007, ISSN 0030-1299

Subject Editor: Esa Ranta, Accepted 18 January 2007

\title{
Reconsidering the mechanistic basis of the metabolic theory of ecology
}

\author{
Michael P. O'Connor, Stanley J. Kemp, Salvatore J. Agosta, Frank Hansen, \\ Annette E. Sieg, Bryan P. Wallace, James N. McNair and Arthur E. Dunham
}

M. P. O'Connor (mike.oconnor@drexel.edu), A. E. Sieg and B. P. Wallace, Dept of Bioscience and Biotechnology, Drexel Univ., Philadelphia, PA 19104, USA. Present address for BPW: Duke Univ. Center for Marine Conservation, Nicholas School for the Environment and Earth Sciences, 135 Duke University Marine Lab Road, Beaufort, NC 28516, USA. - S. J. Kemp, S. J. Agosta, F. Hansen and A. E. Dunham, Dept of Biology, Univ. of Pennsylvania, 415 S. University Avenue, Philadelphia, PA 19104, USA. Present address for SJK: Dept of Biology, Pennsylvania State Univ., Berks Campus, Tulpehocken Rd., Reading, PA 19610, USA. - J. N. McNair, Patrick Center for Environmental Research, Academy of Natural Sciences of Philadelphia, 1900 Benjamin Franklin Parkway, Philadelphia, PA 19103, USA

\begin{abstract}
The recently proposed metabolic theory of ecology (MTE) claims to provide a mechanistic explanation for long known allometric relationships between mass and metabolic rate. The MTE postulates that these patterns of allometry are driven by the primary selective constraint of transport of energy and materials. However, recent evidence along several different lines has called into question both the adequacy and the universality of this mechanism. We review the accumulating body of literature on this subject, adding our own concerns and criticisms. In addition to other difficulties, we argue that MTE fails as a mechanistic explanation of mass versus metabolic rate allometries because: 1) circulatory cost minimization is not a tenable criterion for evolutionary optimization, 2) the Boltzmann type relationships on which MTE depends are inadequate descriptors of complex metabolic pathways, and 3) most of the hypotheses advanced by the MTE do not, in fact, depend on the proposed mechanism and therefore cannot be used to test the theory. We conclude that the MTE should be abandoned as a monolithic explanation for allometric patterns, and that a more realistic path toward a better understanding of allometry would be to consider multiple explanatory mechanisms for physiological allometries.
\end{abstract}

Biologists have been intrigued by allometric scaling relationships between organismal form, function, and ecology for more than a century. Such scaling relationships are taxonomically pervasive and have inspired a literature that, by 20 years ago, resulted in three nearly simultaneous, but remarkably different, book-length reviews (Peters 1983, Calder 1984, Schmidt-Nielsen 1984). Nonetheless, there is currently no widely accepted general mechanism for these patterns. Hypothesized explanations include structural and functional factors (e.g. surface area/volume effects on exchanges of heat and metabolites; reviewed by Calder 1984, Dodds et al. 2001), biomechanical requirements for support and fracture resistance (McMahon 1973, Biewener 2005), and correlated natural selection on body size and life history (Kozlowski and Weiner 1997).

Recently, West et al. and others have advanced a metabolic theory of ecology (MTE - West et al. 1997, 1999, Gillooly et al. 2001, 2002, Brown et al. 2004a) which:

1. conjectures that a quasi-fractal structure of distributional networks like circulatory systems requires an allometry (e.g. metabolism $=\alpha$ mass $^{\beta}$, where $\alpha$ is a "normalization constant" and $\beta$ is a scaling exponent) in order to minimize transport costs of energy and materials

2. argues that minimizing these transport costs requires a scaling exponent of $\beta=3 / 4$, which in 
turn, is the basis of scaling exponents for various other properties (e.g. development time, longevity, community level properties) that are multiples of $1 / 4$

3. links metabolism and temperature via the Boltzmann factor used to predict the kinetics of simple biomolecular reactions

The proposed mechanistic explanation has energized the consideration of allometric effects in ecology by the original authors (summarized in Brown et al. 2004a) and others (Cottingham and Zens 2004, Jetz et al. 2004, Kaspari 2004, Marquet et al. 2004), producing a number of substantial hypotheses.

However, there is considerable debate in the literature regarding the support for predictions of the MTE as well as the validity of the theory's underlying assumptions. Criticism has focused on a variety of issues, including deviations of allometric scaling exponents from the proposed quarter power rule (Dodds et al. 2001, White and Seymour 2003, 2005, Savage et al. 2004, Reich et al. 2006, Muller-Landau et al. 2006), other hypotheses which may account for the observed allometries (Kozlowski and Weiner 1997, Darveau et al. 2002, Demetrius 2003, Hochachka et al. 2003, Bejan 2005), the validity of the assumptions regarding circulatory systems (Fung 1997, Kozlowski and Konarzewski 2003, Dawson 2005), the inadequacy of Boltzmann factors for modeling dynamics of complex metabolic pathways (Darveau et al. 2002, Hochachka et al. 2003, Cyr and Walker 2004, Marquet et al. 2004, Clarke 2004), the limited scope of the predictive capacity of MTE (Cyr and Pace 1993, Tilman et al. 2004), internal inconsistency of the theory (Makarieva et al. 2005), and the epistemological difficulties associated with conducting an adequate test of the theory itself (Cyr and Walker 2004, Koehl and Wolcott 2004, Marquet et al. 2004, Tilman et al. 2004).

MTE is advanced on two major philosophical premises: 1) that allometric phenomena require a mechanistic explanation and that MTE provides that mechanism based on physical and physiological first principles, and 2) that the provided mechanism motivates and justifies renewed and expanded work on the ecological implications of allometry. We begin by discussing three general philosophical issues with the MTE (mechanisms and models, first principles and the MTE, and the fact that the MTE and its hypotheses are dissociated). We then discuss four key mechanistic issues with the bases of MTE (distributive networks and allometric optimization; Boltzmann relationships, temperature, and metabolism; the assumption that metabolic allometry is causal to other allometries; and the plausibility of a single explanatory mechanism for allometry). Finally, we outline what we consider to be a better research path for moving forward in understanding allometry, and we end by summarizing our main conclusions.

\section{General philosophical issues with the MTE}

\section{Mechanisms and models}

A central question to be resolved is whether the explanation of allometric relationships that the MTE provides is actually mechanistic. We argue that one should distinguish three different types of explanatory models. The first type - phenomenological - requires an empirical, but not a mechanistic, relationship between two variables. Classical regressions of metabolic rate on mass fall into this category (Peters 1983, 1991, Calder 1984, Schmidt-Nielsen 1984). An underlying possibility of all such models is that each of the variables may depend on unanalyzed (hidden) variables that create the pattern. Methods used in creating such models are typically statistical in nature. The models are subject to the limitation that extrapolation beyond the measured data or application to systems in which the underlying variables may differ is always suspect (Calder 1984). Deviations from predictions are part of the pattern, are assumed to be due to variation in underlying variables or 'random' biological variation, and are treated as data for further analyses.

In the second type of model - mechanistic - the input variables are linked to the output variables and model predictions by a series of causal relationships. The causal mechanisms can be either well accepted or hypothetical, and the quality of the predictions is judged by the plausibility and importance of the hypothesized mechanisms in the system considered. A number of classical models in both biophysical ecology (Gates 1980, Vogel 2000) and population ecology (Gotelli 2001) fall into this category. A notable characteristic of such models is the need for simplifying assumptions (ignoring real biological complications and other operative processes) to allow the causal linkages to be made. Deviations from predictions (other than those due to variability in measurements) may be attributed to operation of ignored processes or failure of the model to adequately describe the processes under consideration. The major question with such deviations is whether they falsify the model or simply indicate that refinements are required. The virtue of such models is that, as long as the underlying mechanisms are well described, changing conditions simply change some of the inputs to the model, rather than potentially invalidating the entire model as occurs with phenomenological models (Dunham and Beaupre 1998). Thus, 
causal models have a robustness and heuristic value not achieved by purely statistical models.

The models advanced in MTE are a compromise between these two types of models. Proxy variables for factors of likely mechanistic importance are used in a statistical framework. For example, degree days represent thermal inputs (Gillooly and Dodson 2001), Boltzmann relationships represent thermal effects on biological processes (Gillooly et al. 2001, 2002, 2006), potential constraints on cardio-respiratory distribution of metabolites in vertebrates represent conjectural constraints in organisms lacking cardiovascular and respiratory systems (West et al. 1997), and presumptive physiological efficiencies serve as proxies for fitness (West et al. 1997, Brown et al. 2004a).

Such analyses have a long and useful history in the physiological explanation of ecological processes (Kingsolver and Watt 1984, Porter et al. 2002). But, the extent to which such regressions provide broadly generalizable mechanisms as thermal environments and physiologies, distributional systems and constraints, and selective forces all vary is an open question. Likewise, the extent to which the linear equations, fitted by regression, constrain the mathematical form of a generalized mechanism is unclear, particularly in systems whose dynamics result from multiple, interacting, non-linear systems.

We argue that most putative MTE mechanisms must be regarded as statistical models based on plausibly mechanistic variables. Dependence on proxy variables such as the activation energy of macroscopic processes (a key component of the MTE, discussed below in connection with Boltzmann relationships and the temperature-dependence of metabolism) (Brown et al. 2004a) is a hallmark of such statistically based models. The minimization of cardiovascular transport costs, modeled primarily on high-pressure mammalian circulatory systems (another key component of the MTE, discussed below in connection with distributive networks and allometric optimization), is a potential mechanistic and falsifiable hypothesis for some animals. However, when it is successively extended to animals with low pressure or open circulatory systems, or even no circulatory systems (Brown et al. 2004a, West and Brown 2005) on the assumption that similar processes and constraints will apply, this hypothesis becomes increasingly non-mechanistic and non-falsifiable. Arbitrarily assigning some components of interspecific allometric variation to the scaling exponent while assigning others to the normalization constant (Savage et al. 2004, West and Brown 2005) is a reification of assumptions of statistical fitting and not a mechanism (as discussed below in connection with the plausibility of a single explanatory mechanism for allometry). Such speculations may ultimately be justified by the elaboration of true mechanisms, but they are statistical models, not mechanisms. They do not possess the robustness and applicability of mechanisms, and cannot be justified by the very patterns to which the statistical models were fit.

\section{First principles and the MTE}

One advantage claimed for MTE is that the theory is based on first principles and depends on fewer assumptions and fitted parameters than other hypothetical systems (West et al. 2003, 2004, Brown et al. 2004a, Harte 2004). Such claims must, however, be clarified by defining first principles, which is rarely, if ever, done. An extensional definition for the MTE appears to include arguments based on fields outside of biology (primarily chemistry and physics, West et al. 1997, Porter et al. 2002, Brown et al. 2004a) or on commonly accepted physiological principles (e.g. costs should be minimized, Brown et al. 2004a).

Our experience, as authors of models based on physical and physiological principles (O’Connor 1999, O'Connor and Dodson 1999, Dunham et al. 1989, O'Connor et al. 2006), is that freedom from assumptions is neither assured nor likely. Well-specified ecological models based on physical laws and relationships have the virtue of depending on processes whose dominant dynamics are well understood, and of clearly outlining the assumptions made, but they do not free one from the need for assumptions. In fact, the application of simple physical principles to ecological systems requires a plethora of assumptions in practice because of the complex series of organizational levels (from macromolecules to ecosystems, potentially) through which physical effects are filtered to reach their ecological effects.

Potentially limiting assumptions of the MTE regarding metabolic allometry include: 1) that metabolic rate is limited primarily by distributional networks (Darveau et al. 2002, Hochachka et al. 2003), even in unicellular organisms (West et al. 1997, Makarieva et al. 2005), 2) that circulatory transport costs will indeed be minimized, 3) that capillary diameters are size invariant (Dawson 2001, 2005), 4) that the simplified description of the circulatory system is adequate (Kozlowski and Konarzewski 2004), even for organisms with open circulatory systems or no cardiovascular systems, 5) that arterial impedance matching is sensitive to the blood volumes, flows, and anatomy adduced by West and Brown (2005), 6) that branching in real bodies sufficiently approximates the simplifying assumptions (Fung 1997) to justify MTE's arguments, and 7) that the "normalization constants" in allometric power equations are unimportant in comparison to the scaling exponents (Brown et al. 2004a). Thus, the MTE is not free from assumptions, and in later sections, we 
argue that several of these assumptions (Gillooly et al. 2001, 2002, Brown et al. 2004a) are untenable.

Characterizing MTE as based on first principles does not mean that it is assumption free. It should mean that the assumptions are easily identifiable and the consequences of violating those assumptions are easily understood. However, it should not otherwise confer special status or logical primacy on MTE as an explanatory mechanism.

\section{MTE and its hypotheses are dissociated}

A surprising aspect of many allometric hypotheses motivated by MTE is that their support is grounded less in the mechanisms proposed by Brown et al. (YEAR?, West et al. 1997, Gillooly et al. 2001, Brown et al. 2004a) than on pre-existing empirical patterns. The proposed mechanisms are logically dissociated from the phenomena they are meant to explain. Neither transport cost minimization nor activation energies are tightly or mechanistically linked to allometric phenomena (Cyr and Walker 2004, Marquet et al. 2004). Rather, they serve as phenomenological descriptors of allometries (Koehl and Wolcott 2004).

Although the proposed mechanisms of MTE encourage hypothesis formation, the allometric hypotheses advanced do not depend logically on those mechanisms (West et al. 1997, Brown et al. 2004a, West and Brown 2005). Instead, the hypotheses depend primarily on phenomenological quarter-power allometries for metabolism, population density, and physiological and ecological times or rates (see synopses in Brown et al. 2004a) which generally antedate MTE (Calder 1984). As an example, consider the assertion that organisms of different sizes and trophic levels process approximately equal energy throughputs in many ecosystems (Brown et al. 2004a). Tilman et al. (2004) note that this hypothesis is among the most original and surprising of the hypotheses proceeding from MTE. The premises necessary to this hypothesis include: 1) the $3 / 4$ power allometry of whole animal metabolism, 2) the $10 \%$ transfer ratio for energy passing from one trophic level to the next (Lindeman 1942), 3) a mass ratio of predators to their prey of $10^{4}$ (Brown et al. 2004a) and 4) an allometry of population density (Calder 1984). Premises 1, 2 and 4 are empirical patterns that entered the literature before MTE, whereas premise 3 is claimed by Brown et al. (2004a) for marine systems, but depends on none of the ascribed mechanisms. Several authors have noted that the mechanistic bases provided by MTE justify such hypotheses (Cyr and Walker 2004, Marquet et al. 2004), but the hypotheses do not actually rest on the mechanisms. This dissociation is not an indictment of the proposed MTE mechanisms. We merely note that the mechanisms are not critical to the hypotheses.

\section{Mechanistic issues with the MTE}

\section{Distributive networks and allometric optimization}

The core assertion of the MTE is that minimizing the cost of transport in distribution networks requires quarter power allometries, which provides the mechanistic basis for understanding these relationships (West et al. 1997, West and Brown 2005). However, we contend that this simple optimization criterion is not sufficient to describe the variety of selective forces that likely operate in determining metabolic rates. First, true optimization of isolated physiological systems (like metabolism) is difficult, if not impossible to achieve in nature, as has recently been reviewed for the closely related concept of metabolic symmorphosis (Dudley and Gans 1991, Gordon 1998, Jones 1998). Pleiotropy, multiple uses for structures, and variable selective environments all constrain optimization of physiological systems. Selection optimizes the expected lifetime reproductive success of entire organisms rather than the efficiency of a particular component of metabolism, although the two may well be correlated. Many aspects of metabolism, active and resting, could conceivably affect the fitness of an organism. Therefore, the idea that natural selection would optimize cardiovascular transport costs (in isolation from other systems), which would then come to dominate metabolic allometry, seems unlikely.

When conflicting demands are placed on a system, biologically optimal solutions are context dependent, and the results of optimality analyses depend on optimization criteria (Taylor 1998). Rarely can any of a set of criteria be confidently identified with the fitness of the organism. Take for example, the differences between the demands placed on circulatory systems at resting and maximal metabolism. In mammals, resting and maximal metabolism subserve related, but different, functions with fluxes of ATP, oxygen, and blood differing over 5-40 fold ranges (Weibel and Hoppeler 2005). Resting and maximal metabolism are dominated by different tissues (predominantly muscle at maximal throughput) with different capillary densities (due to recruitment primarily in muscle) and metabolic pathways (Cameron 1989, Weibel and Hoppeler 2005). Savage et al. (2004) provided strong evidence of different allometric scaling when maximal and resting metabolic rates were considered. Different metabolic rates and circulatory patterns would be optimized sensu West et al. (1997) by different body sizes and cardiovascular structures. Despite statistical, and likely 
mechanistic, linkages between resting and maximal metabolism in different mammals, it is difficult to argue that either extreme is more closely related to fitness. Further, it is difficult to determine what the optimal compromise might be, even when the proposed mechanism is rather simple (Jones 1998). Similarly, organisms might benefit from minimizing metabolic expenditures or from maximizing activity, with its attendant increase in metabolic expenditures. Discerning the optimal compromise is difficult, and within tetrapods, the continued existence of relatively bradymetabolic ectotherms and comparatively tachymetabolic endotherms argues that multiple, clade specific optima are possible (Pough 1980). As more variables are considered, optimization and allocation problems become less tractable, with broader sets of solutions (Niewiarowski and Dunham 1994). The degree to which minimizing a subset of costs of metabolite transport determines metabolic rate needs further attention.

To whatever extent selection might optimize bulk transport via distributional networks, the specific mechanism proposed for MTE (isolated minimization of fluid transport costs) is a poor subject for minimization because it is unlikely that transport cost maps in a uniform or monotonic manner onto fitness (although exactly how these are related is typically unknown). Because selection optimizes organismal fitness, optimal design hypotheses must consider both costs and benefits (Pyke 1984, Weibel et al. 1998). Fluid transport costs are truly minimized only if the costs of transport, blood flow rate, and the supported metabolism are all zero, which is clearly not feasible. The benefits of transport, in this case, are the physiological and behavioral actions possible at a given blood flow, i.e. what the flows make possible. Consider the example of high output shock from clinical physiology (Guyton and Hall 2001, Berne et al. 2004). For any of several reasons, but often due to infections, the smooth muscles in arterioles in numerous vascular beds relax, and substantially reduce the systemic vascular resistance. As resistance falls, so does arterial blood pressure and blood flow rates in critical vascular beds, including those above the heart that require elevated blood pressure to maintain perfusion. Healthy hearts respond by increasing heart rate, stroke volume, and total cardiac output. The lowered resistance (and cost of transport per unit blood flow) is clearly within the physiological capabilities of the cardiovascular system, but is pathological rather than adaptive. This example is extreme, but it points out the pitfalls of unconstrained minimization of transport costs. What is actually required to maximize fitness is a simultaneous optimization of both costs and benefits. This optimization would require that both costs and benefits be expressed in a common currency, fitness being the logical candidate. Unfortunately, the conver- sions of either transport costs or the supported capabilities to fitness are unknown at present. We would expect selection to minimize transport costs, all other things being equal. But other things (e.g. benefits of transport) are not necessarily equal. Elegant mathematical analyses of the wrong optimization criteria may lead to erroneous conclusions.

If the marginal energetic costs of transport due to the architecture of the vessels were a large fraction of the organism's metabolic rate, one might still argue that unconstrained minimization of costs would be biologically reasonable. However, using allometric expressions for metabolism, cardiac output and blood pressure (Calder 1984, Li 2000, Dawson 2001, 2005), we estimate that the total cost of cardiac work is less than $8 \%$ of resting metabolism for mammals. What fraction of this energetic cost can be attributed to deviation from West et al.'s (1997) optimal geometry is unclear, but these costs cannot come to dominate an animal's energy budget. While it is true that energy allocated towards circulatory needs decreases the energy available for growth and reproduction, to assume that selective forces solely minimize these circulatory costs is to disregard the numerous constraints imposed by other aspects of natural selection.

Finally, the assumption that fractal distributional networks both support and constrain metabolism in unicells (West et al. 1997) is incorrect and cannot be regarded as mechanistic. Although transmembrane transporters are involved in the transport of metabolic fuels, the transport of oxygen and carbon dioxide in unicells (and in the cells of metazoans) is diffusive and is not based on bulk flow in defined pathways (Cameron 1989, Vogel 1994, Berne et al. 2004). The transport of respiratory gases is the rate-limiting step for circulatory support of metabolism in vertebrates and led to the development of one of the predominant hypotheses of symmorphosis (Taylor and Wiebel 1981, Taylor et al. 1981, Weibel et al. 1998). Perhaps diffusion pathways for respiratory gases within cells should be treated as fractal networks. Perhaps distributional networks for other molecules based on microtubules and motor proteins meet similar requirements, but there is no evidence that such networks function as mechanisms enforcing quarter power allometries in unicells. It is highly unlikely that diffusive transport of oxygen and fuels in unicells can be described by kinetics similar to those in large animals with circulatory transport. Not surprisingly, recent evidence shows that predictions made by the MTE regarding single cells are poorly supported by data (Makarieva et al. 2005).

Emprical evidence disputing the main predictions of the MTE with regards to the mechanism of metabolic scaling and vascular transport systems is mounting. Recent studies of metabolic scaling in plants indicate a substantial deviation from the predictions of 
MTE (Reich et al. 2006). West et al. (1997, Brown et al. 2005) asserted that the MTE would apply to plants owing to their comparable fluid and nutrient distribution network (xylem and phloem). However, the empirical results of Reich et al. (2006) and others (Muller-Landau et al. 2006) now indicate that MTE predictions fail in vascular plants (Makarieva et al. 2005). This is fairly strong evidence against the original claim of the universality of the mechanism proposed by MTE to explain metabolic allometries.

We do not argue that transport costs in quasi-fractal networks cannot be important in structuring vascular systems and metabolism over sufficiently broad ranges of body size. It is certainly conceivable that over very broad size ranges, vascular systems sufficiently divergent from the structure required by MTE (West et al. 1997, West and Brown 2005) will result in poor circulatory function and provide a basis for selection of organisms more in line with its requirements. However, it is not clear what level of divergence from the MTE model would result in cost-based effects on fitness that overcome the associated benefits of the circulation.

\section{Boltzmann relationships, temperature, and metabolism}

Although most components of metabolism are affected by temperature, proposing Boltzmann-type relationships as the mechanism for the temperature dependence of metabolism is untenable. First, no macroscopic equivalent of the activation energy cited by Gillooly et al. $(2001,2002)$ exists. For sufficiently simple reactions, the activation energy has a real, if approximate, referent (i.e. the difference in free energy between the reactants and a "high energy" intermediate) that becomes more approximate in biochemical pathways, though still widely used (Bentz 1992, Chan and Dill 1998, Clarke 2004). In simple biochemical pathways with a single rate limiting step, free of allosteric controls, one could argue that the activation energy for the rate limiting reaction would be an adequate descriptor at least within the temperature range in which enzyme activity was not strongly affected by temperatures.

Alternatively, Clarke (2004) argues that enzymes substantially change kinetic constraints making diffusion and the creation and breaking of weak molecular bonds the dominant rate limiting effects. In either case, at the level of the cell with its complex, feedback controlled network of reactions with numerous metabolic checkpoints, each of which responds to several controllers and external conditions, no equivalent of the activation energy exists (Hochachka et al. 2003). Further, at this level, the simplicity of control implied by the Boltzmann relationship does not exist (Rolfe and
Brown 1997, Darveau et al. 2002, 2003, Hochachka et al. 2003). As one considers organ systems, individuals, population, and ecosystem levels of organization, the complexity and diversity of the organization networks and their responses to temperature all increase progressively. Any putative relationship based on activation energies for processes at these different levels of organization (e.g. for life history events) can be, at best, an observed correlation with temperature, and not a mechanism.

Brown et al. (2004a), in posing the hypothesized MTE mechanisms, suggest that the multiplicity of levels of biological organization and explanation make mechanism a slippery concept, noting that "one person's mechanism is another's empirical phenomenology". We reject this relativism and argue, instead, that the explained phenomenon and the proposed mechanisms themselves determine the critical levels of organization where the mechanics underlying phenomena must be clear. Mechanisms demand causational reduction adequate to describe the target phenomena both qualitatively and quantitatively (Dunham and Beaupre 1998). Causational reduction requires a stepby-step, cogs and gears connection between the dependent effect and the causative process. By this definition, correlative relationships like the proposed MTE bases cannot be regarded as mechanistic. MTE does pose the mathematical forms, but as we argue above, it lacks the causative linkage from the mechanisms (here, a molecular level activation energy) to the thermal dependence of whole animal metabolism and life history events.

Furthermore, agreement of empirical data with the form of a Boltzmann relationship does not justify the promotion of a correlation to a mechanism. First, even tight correlation does not constitute causation. Second, as Brown et al. (2004a) note, a Boltzmann relationship between metabolism and temperature results in fairly small errors when fitted to a traditional exponential $\left(Q_{10}\right)$ relationship, particularly with a fitted value of activation energy that minimizes those errors. Hence two different mathematical bases (Boltzmann and exponential) with similar predictions, but potentially different underlying mechanisms, would both produce small errors when compared with empirical data. Reduction of residual errors by regression does not validate a mechanism.

Another reservation about the Boltzmann relationships arises from a poor fit to thermal physiology, particularly the short and long term variation of metabolism in response to changes in body temperature. Here, we concentrate on responses in ectothermic animals, but similar arguments can be made for endotherms. The literature of acclimation, particularly of acclimation of metabolic rates to varying temperatures in ectotherms is extensive and taxonomically diverse (Beyer and Spotila 1994, Guderley and St. Pierre 1996, 
Gracey et al. 2004, Lesser and Kruse 2004, Zakhartsev et al. 2004, Whiteley and Faulkner 2005). The typical pattern is that an ectotherm held at one temperature, then subjected to sudden temperature changes, might experience changes in metabolic rate compatible with a Boltzmann or $\mathrm{Q}_{10}$ relationship (Fig. 1, $33^{\circ} \mathrm{C}$ acclimation). That same animal, after being held at a new temperature (Fig. $1,16^{\circ} \mathrm{C}$ ) for days to weeks might show a similar but shifted response such that the difference in metabolic rate at each experiment's acclimation temperature is smaller than would be expected from the acute responses (Hill et al. 2004). Thus, the magnitude of the metabolic response depends on the timing of the temperature change. This cannot be accounted for by a change in normalization constant, as claimed by Gillooly et al. (2006). The extent of compensation varies from practically nil (the temperature response curves at the two acclimation temperatures are virtually superimposable) to perfect compensation (metabolic rate at $T_{1}$ when acclimated to $T_{1}$ equals metabolic rate at $T_{2}$ when acclimated to $T_{2}$ ) with a few organisms even showing over-compensation. The dependence of metabolism on temperature is both subject to selection among animals and physiologically adjustable within a single organism. This variation is inconsistent with a Boltzmann relation that can be used to predict thermal variations in metabolic rates in individuals, populations, species, higher taxa, and even whole ecosystems.

We do not argue that temperature does not affect metabolism, but that the proposed Boltzmann relation cannot encompass the patterns of variation seen

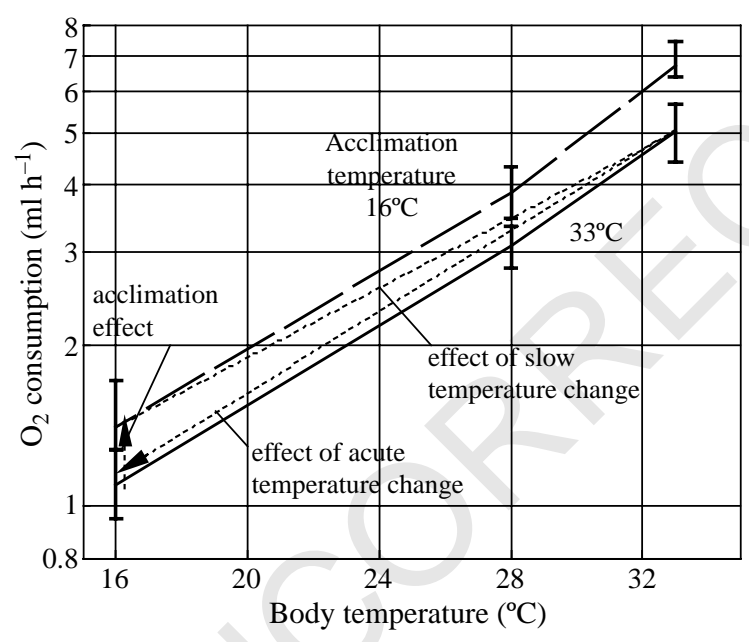

Fig. 1. Metabolic rates of Sceloporus occidentalis acclimated to either 16 or $33^{\circ} \mathrm{C}$ for 5 weeks. Acclimation effect partially compensates for change in temperature and would decrease the estimated dependence of metabolism on temperature. Oxygen consumption rate is shown on log scale to "straighten" relationships. Data from Dawson and Bartholomew 1956, diagram modified from Hill et al. 2004. commonly in nature. Alternative, but less monolithic, mechanisms have been proposed to account for the variation of metabolism with temperature (Clarke 2004, Clarke and Fraser 2004).

\section{Metabolic allometry is causal to other allometries}

A critical assumption of MTE is that allometries for other organismal (e.g. heart rate, development time, longevity) and population (e.g. density) traits depend on metabolism. From this, it follows that if metabolic scaling is explained mechanistically, then allometries for these traits are also explained (Brown et al. 2004a). To the best of our knowledge, no data or theory adequately tests this assumption. Although some factors are necessarily related to metabolism (e.g. mass specific metabolic rate), and others are plausibly related (e.g. heart rate, population metabolic demand), we have two general concerns related to this assumption in the context of MTE.

First, vertebrate (particularly mammalian) cardiovascular allometries are a curious mix of traits, some of which scale allometrically according to quarter power laws (e.g. heart rate), some of which appear nearly invariant (mean arterial blood pressure), and some of which scale with other exponents (e.g. pulmonary and, possibly, systemic capillary diameter-Li 2000, Dawson 2001, 2005). Recent work also suggests that metabolism scales differently in mammals depending on activity, with basal, field, and maximal aerobic metabolism scaling with progressively steeper exponents (White and Seymour 2003, 2005, Savage et al. 2004). In plants, predictions of MTE regarding scaling of allometries in tropical forests (growth and mortality with size) were categorically not met in an analysis of a global data set (Muller-Landau et al. 2006). Whether some of these deviations from MTE predictions are statistical artifacts, simple biomechanical results, or reflections of a deeper complexity and heterogeneity remains unresolved at this time. However, since the same sources of error exist in all allometric data sets, statistical artifacts cannot only apply when the predictions of the MTE do not match the data.

Second, a familiar counterexample suggests that allometries for different traits may be independently selected, counter to predictions of the MTE. Differences in metabolic rates among breeds of domestic dogs follow approximately the classical quarter power allometry (Speakman et al. 2003). However, the interbreed allometry for longevity is the inverse of the classical pattern with small breeds being generally longer-lived than large breeds (Patronek et al. 1997, Speakman et al. 2003, Austad 2005). In this case, the allometries, like the breeds themselves, are the product of artificial selection, and some longevity effects may be due to 
inbreeding. Nonetheless, the patterns suggest that selection (albeit artificial) can lead to starkly different effects on allometries for different characters. Recent work documents similar patterns in mice and Drosophila and suggests a mitochondrial mechanism for elevating metabolic rates while protecting against free radicals and extending life span ( $\mathrm{Tu}$ and Tatar 2003, Speakman et al. 2004, Van Voorhies 2004). These examples come from organisms that have been subject to artificial selection and are, hence, suspect. Glazier (2005), however, documents a variety of intraspecific metabolic allometries: 1) many with allometric exponents differing substantially from 0.75 , and 2) many that are poorly described by linear relationships between the logarithms of mass and metabolism.

\section{Plausibility of a single explanatory mechanism for allometry}

Another proposed advantage for MTE is that a single mechanism (minimization of transport costs in distributional networks) accounts for quarter power allometries for metabolism, extending from the smallest microorganisms to the largest whales (Brown et al. 2004a, West and Brown 2005). The presumption is that because an approximate $3 / 4$ power allometry for metabolism extends across such a broad range of organisms, a single (physiological) mechanism should explain the allometry. Furthermore, it is argued that because a power law appears to describe the allometry, the mechanism should lead to a simple power law expression (West et al. 2003). This argument is flawed for several reasons. First, metabolic allometries from microorganisms to whales do not clearly reflect a single relationship. Even if we ignore the variability of metabolic rates with activity level, the potentially different scaling exponents of resting and non-resting metabolism in mammals (White and Seymour 2005), the range of reported allometric exponents (Calder 1984, Dodds et al. 2001, White and Seymour 2005), and the diversity of normalization constants (i.e. the resting metabolic rate at a standard mass) all raise questions about the proposed universal mechanisms. Variation in normalization constants has been attributed to phylogeny (Demetrius 2003, Hochachka et al. 2003, Brown et al. 2004a). At minimum, such variation in normalization constants suggests that factors unrelated to allometric constraints can affect a taxon's mass, metabolic rate, or both. Also, the physics and physiology of metabolite transport, heat transfer, and biomechanics differ substantially in large versus small animals. Large animals exist in high Reynolds number environments dominated by inertial forces, and require bulk transport systems to transport metabolites. Small unicells, on the other hand, inhabit low Reynolds number environments dominated by viscous forces and possess diffusive metabolite transfer (Schmidt-Nielsen 1984, Vogel 1994, 2000). Counter to the predictions of MTE, Makarieva et al. (2005), trying to control for differences due to culture conditions, found little difference in minimal and maximal mass-specific metabolic rates in a variety of microorganisms with cell volumes differing by a million fold, but found considerable variation in metabolic rates among taxa at a single size. Thus, allometric mechanisms that focus on metabolite transport to and from surfaces, biomechanical constraints on structures (McMahon 1973, 1975, Biewener 2005), or bulk flow distribution networks (West et al. 1997, Brown et al. 2004a) will likely work quite differently in microorganisms and whales (Table 1).

Two important qualifiers are often applied to MTE's arguments. First some argue that MTE's mechanisms are meant to explain global allometric patterns, i.e. those applying over broad ranges of body masses, rather than the fine tuning of differences among animals that are relatively close in size (Brown et al. 2004a, West and Brown 2005). Second, Brown et al. (2004a, b), Savage et al. 2004, West and Brown 2005) have argued that we should distinguish two types of allometric variation in traits: within-clade variation that is explained by MTE's mechanisms and affects the allometric exponents and between-clade variation related to other factors that primarily affects the normalization constant.

Aside from their ad hoc nature, these qualifiers create a series of conceptual problems. It is certainly possible that, over sufficiently broad mass scales, the processes presented by West et al. (1997) could enforce a level of conformance to an allometry that other factors could obscure at finer scales. But the requirement for longer mass scales severely limits the applicability of MTE to ecology (Tilman et al. 2004). Furthermore, extending over very large mass scales increases the likelihood that among-clade differences in biology will arise, increasing the import of other factors (e.g. open vs closed circulatory systems) and differences in normalization constants. Indeed, one of the problems with MTE as a universal mechanism is that extension to broader mass scales requires one to focus increasingly on exponents within clades and ignore variation in normalization constants (Brown and West 2005). A second problem is that distinguishing between effects on allometric exponents and normalization constants reifies a regression result as the mathematical form of a physical effect. Further it assumes that selective variation e.g. in metabolic rate, is neatly divisible into separate selective forces that apply to the slope or to the intercept of that regression, without supporting data. Finally, both of these qualifiers make the point that physiological effects other than the putative MTE mechanisms are critically important in setting animal 
Table 1. Summary of hypothetical ultimate mechanisms to account for metabolic and other allometries.

\begin{tabular}{|c|c|c|c|c|}
\hline Potential mechanism & Proponents & Idea & Works well for & Limitation \\
\hline $\begin{array}{l}\text { Surface area/volume ratios } \\
2 / 3 \text { allometry }\end{array}$ & $\begin{array}{l}\text { Numerous classical; Dawson 2001, } \\
\text { Dodds et al. } 2001\end{array}$ & $\begin{array}{l}\text { for conserved quantities/resources: } \\
\text { - exchanges proportional to surface } \\
\text { area } \\
\text { - demand, reserves-proportional to } \\
\text { mass }\end{array}$ & $\begin{array}{l}\text { - large organisms with rate-limiting } \\
\text { exchanges across skin e.g. heat. } \\
\text { - smaller organisms with similar } \\
\text { exchanges e.g. metabolites. }\end{array}$ & $\begin{array}{l}\text { - are exchanges rate limiting? } \\
\text { - are allometries for large and small } \\
\text { animals similar? } \\
\text { - adaptations for exchange can } \\
\text { change allometries }\end{array}$ \\
\hline $\begin{array}{l}\text { Biomechanics - elastic } \\
\text { similarity } 3 / 4 \text { allometry }\end{array}$ & McMahon 1973, Biewener 2005 & $\begin{array}{l}\text { structural allometries required for } \\
\text { support in larger organisms }\end{array}$ & $\begin{array}{l}\text { - large vertebrates and invertebrates } \\
\text { - large plants }\end{array}$ & $\begin{array}{l}\text { - smaller organisms } \\
\text { - connection to function e.g. } \\
\text { metabolism.? }\end{array}$ \\
\hline $\begin{array}{l}\text { Distributional networks } \\
3 / 4 \text { allometry }\end{array}$ & $\begin{array}{l}\text { West et al. 1997, Brown et al. } \\
\text { 2004a, Banavar et al. } 1999\end{array}$ & $\begin{array}{l}\text { minimizing cost of transport in } \\
\text { distributional networks }\end{array}$ & $\begin{array}{l}\text { - endotherms with } 3 / 4 \text { power } \\
\text { allometry }\end{array}$ & $\begin{array}{l}\text { - micro-organisms with diffusive } \\
\text { transport } \\
\text { - organisms with different networks } \\
\text { invertebrates? } \\
\text { - fitness effects of failure to } \\
\text { minimize? }\end{array}$ \\
\hline $\begin{array}{l}\text { Life history-size } \\
\text { covariation }\end{array}$ & $\begin{array}{l}\text { Kozlowski and Weiner 1997, } \\
\text { Kozlowski 2000, Speakman } 2005\end{array}$ & $\begin{array}{l}\text { selection on life history phenotype } \\
\text { modifies allometries }\end{array}$ & - theoretical & $\begin{array}{l}\text { - requires pre-existing allometries } \\
\text { - restrictive assumptions }\end{array}$ \\
\hline Quantum statistics & Demetrius 2003 & $\begin{array}{l}\text { constraints on oxidative electron } \\
\text { transport/ATP synthesis-interaction } \\
\text { with life history }\end{array}$ & - theoretical & - restrictive assumptions? \\
\hline
\end{tabular}


characteristics. Factors affecting allometric exponents, i.e. those attributable to MTE mechanisms, are characterized as systematic with broad taxonomic application, while those affecting normalization constants are portrayed as a series of special cases with narrow application (Savage et al. 2004, West and Brown 2005).

Consider for example, the nearly parallel allometries for resting metabolism in mammals and reptiles (Calder 1984). Classically, for animals at similar temperatures, mammals were considered to have metabolic rates 4-10 times those of similar sized reptiles (Kleiber 1961, Bennett and Dawson 1976). Field metabolic rates measured by doubly labeled water techniques differ by 12-20 fold (Nagy 2005). In classic analyses, Hulbert and Else (1981) demonstrated differences in cellular metabolic substrates (e.g. mitochondrial densities) between mammals and reptiles, and Pough (1980) argued that endotherms and ectotherms represented high and low energy throughput strategies for exploiting resources. This suggests that multiple metabolic optima are possible at a given mass. One could argue that multiple optima are consistent with the MTE hypotheses (e.g. via different capillary sizes), but these two strategies demonstrate that multiple mechanisms control metabolic rate at different body sizes and that metabolic allometries are affected differently in mammals and reptiles.

Finally, Occam's razor is invoked to support a single causal mechanism for widespread allometries (West and Brown 2005). The claim is that given the universality of the $3 / 4$ power allometry, a single causal mechanism is more parsimonious than several. First, we should note that logical parsimony is a human preference rather than a natural law (Webb 1996, Bensusan 1998). More importantly, the claimed universality of a simple $3 / 4$ power allometry or its causal mechanism is far from obvious whether one considers the numerical value of the exponents, the range of body sizes over which the allometry extends, or variation among taxa at particular size ranges (Reich et al. 2004, Muller-Landau et al. 2006).

\section{Moving forward in understanding allometry}

We believe it to be unfortunate, and counterproductive, that mechanistic explanations of metabolic allometries are often treated as being mutually exclusive, so that at most one can be correct (Banavar et al. 2003, Darveau et al. 2003, West et al. 2003). For a number of reasons, we contend that allometric scaling is most likely the product of more than one mechanism, with each mechanism playing a different role under different circumstances.

Proposed explanations for allometries fall into two broad classes (Table 1,2). Ultimate mechanisms seek to explain the evolutionary "why" of allometries (Table 1). They invoke a fitness benefit, often based on physical mechanisms (McMahon 1973, West et al. 1997, Demetrius 2003), or selective mechanisms (Kozlowski and Weiner 1997) to create an allometry. Ultimate hypotheses also tend to specify selected allometric exponents (McMahon 1973, West et al. 1997, Dodds et al. 2001, Demetrius 2003) or indicate changes in allometric exponents (Kozlowski and Weiner 1997).

More proximate mechanisms, on the other hand, seek to explain the cellular or biochemical mechanisms by which allometric control of metabolism operates (Table 2). Proximate mechanisms specify how allometric variation in metabolism can be achieved by varying cellular structures or functions, but do not specify allometric exponents. Whichever ultimate mechanism one favors, selection will need to enforce the allometry via one of the more proximate mechanisms. Thus, sets of ultimate and proximate mechanisms must work in series and cannot be mutually exclusive.

If we concentrate on the more ultimate proposed mechanisms (Table 1), none of the hypotheses clearly extends to all organisms. Surface-area-to-volume hypotheses depend on demonstrations that particular heat or resource budgets constrain metabolism (Hochachka et al. 2003). Varying internal and external exchange physiologies and exchangers with changes in body size (Schmidt-Nielsen 1984, Vogel 1994, 2000, O'Connor and Dodson 1999) suggest that different types of exchanges (heat, resources) will be constraining at different sizes. Whether different exchanges would produce a homogeneous allometry needs investigation. Elastic similarity (McMahon 1973) modifies the surface area to volume ratio expected under geometric similarity, but otherwise suffers the same constraints mentioned above. We have already discussed some limitations on MTE-like bulk flow transport hypotheses (West et al. 1997, Banavar et al. 1999). Mechanisms citing selection on life history phenotypes (Kozlowski and Weiner 1997, Demetrius 2003) are highly theoretical and require restrictive assumptions to facilitate their mathematical development. All of these hypotheses depend on optimizations, requiring extrema of physiological flows, costs, or life history characteristics. Such optimizations are rarely unconstrained in biology (Pierce and Ollason 1987, Dudley and Gans 1991, Gordon 1998).

If none of the hypotheses is sufficient alone, neither are their mechanisms, per force, functionally incompatible. Nothing would prevent biomechanical constraints from setting body proportions with their resultant surface to volume ratios (McMahon 1973), and a combination of superficial exchanges (Dodds et al. 2001), internal transport processes (Brown et al. 2004a), and proton and electron motive forces (Demetrius 2003) from acting simultaneously to 
Table 2. Summary of hypothetical proximate mechanisms to account for metabolic and other allometries.

\begin{tabular}{lll}
\hline Potential mechanism & \multicolumn{1}{c}{ Proponents } & \multicolumn{1}{c}{ Idea } \\
\hline Allometric cascades & $\begin{array}{l}\text { Darveau et al. 2002, Hochachka et al. 2003, } \\
\text { Suarez and Darveau 2005 } \\
\text { Jeneson et al. 2000, Moyes and LeMoine 2005, }\end{array}$ & $\begin{array}{l}\text { multiple control points blend allometries at } \\
\text { each control point } \\
\text { control of particular pathways or sets of } \\
\text { pathways }\end{array}$ \\
$\begin{array}{c}\text { Cellular metabolic } \\
\text { substrates }\end{array}$ & $\begin{array}{l}\text { Chaui-Berlinck et al. 2005 } \\
\text { Else and Hulbert 1985, Hulbert and Else 2000, }\end{array}$ & $\begin{array}{l}\text { Adjustment of membrane or mitochondrial } \\
\text { densities and/or properties drives allometric } \\
\text { variation in metabolism }\end{array}$ \\
\hline
\end{tabular}

influence metabolic rates in a group of organisms. Finally, interspecific allometries might be affected by life history selection (Kozlowski and Weiner 1997, Demetrius 2003). Such simultaneous action of different mechanisms might prevent any particular organism from reaching the optimum specified in a particular hypothesis, but that would hardly be surprising (Gould and Lewontin 1979, Pyke 1984, Huey and Bennett 1987, Dudley and Gans 1991).

Perhaps more important is the possibility that multiple mechanisms might be active and produce similar allometries at different body sizes. Thus, surface exchanges (Dodds et al. 2001, Demetrius 2003) of metabolic fuels and gases might affect metabolism more strongly in small organisms, with internal bulk transport and biomechanics (McMahon 1973, West et al. 1997) being more important in larger organisms, and selection on life history strategies (Kozlowski and Weiner 1997) adjusting scaling exponents. Such a multifactorial process would likely produce a series of similar allometries in different taxa at various ranges of body size, much like what one actually finds (Fig. 1 in Calder 1987, Fig. 6 in Martin et al. 2005, Fig. 2 in West and Brown 2005). In any case, numerous points of evidence indicate that multiple processes affect the allometric scaling of metabolism, and acknowledging this will lead to a more realistic understanding of metabolic allometries.

\section{Conclusions}

MTE has been justly praised for reinvigorating the study of metabolic and other allometries in ecology (Cottingham and Zens 2004, Harte 2004, Kaspari 2004). However, the proposed mechanistic bases of $\overline{\mathrm{MTE}}$ are, at minimum, inadequately tested and supported, and are inconsistent with fundamental aspects of ecology and physiology. The cost of transport minimization is an untenable form of optimization by natural selection, and the Boltzmann normalization of thermal effects on metabolism requires a uniform response to temperature simply not observed in animals. We feel that the MTE must be rejected as a solitary mechanism underlying metabolic allometries due to these concerns and others detailed in this paper and elsewhere. While the physical constraints invoked by the MTE may play a role in determining metabolic allometries, problems with the proposed mechanisms as well as the low predictive scope of MTE refute the contention that it successfully explains long-described allometries mechanistically.

Our fundamental problem with MTE's mechanistically based allometry (other than the inconsistencies with ecology and physiology indicated above) is that those mechanisms are treated as presumptively validated. Further, because they are based on first principles, they are assumed to be taxonomically universal, and logically superior to and mutually exclusive of other mechanisms proposed to explain allometries (West and Brown 2003, Brown et al. 2004a, b). We find MTE lacking as a universal explanatory mechanism for allometric scaling because the proposed mechanisms are disconnected from the hypotheses they motivate, the putative mechanisms (minimization of transport costs, population level Boltzmann relationships) are biologically implausible, and the proposed universality of the mechanisms is untenable.

Our purpose here is to argue for a less restrictive view of physiological and life history allometries that allows for the simultaneous operation of multiple mechanisms (Table 1,2). This approach has several benefits. First, it provides a paradigm in which multiple processes can jointly affect allometric relations. Such a paradigm more easily accounts for the variation in both allometric exponents and normalization constants, promoting them from evolutionary noise around a dominant mechanism to potentially interesting biological problems (e.g. why do mammals and reptiles appear to represent alternate fitness optima?). Second, this approach enriches allometric theory by benefiting from the advantages of various hypotheses. MTE has the singular virtue of tying thermal effects to allometric effects, even if we prefer Clarke's (2004- discussed above in "Boltzmann relationships, temperature, and metabolism") mechanism for temperature effects to that of Gillooly et al. (2001). Biomechanical considerations (McMahon 1973, Biewener 2005), although less tightly linked to metabolism than to structural biology, 
produce allometric effects that cannot fail to affect metabolic and other allometries. Life history selection (Kozlowski and Weiner 1997, Kozlowski 2000) focuses attention on the possibly different evolutionary mechanisms by which intraspecific (classical selection) and interspecific (radiation) allometries arise, and on the meaning of those differences for ecology (Kozlowski 2000). Demetrius (2003) makes interesting and potentially testable predictions regarding the dependence of allometry on activity and life history phenotype (weedy species vs those typically in a more crowded competitive environment). Third, a more inclusive approach would shift attention from proving that a particular allometric mechanism is correct, to considering how and under what circumstances various mechanisms might affect allometry. Ecology has, in the past, learned the disservice done by trying to prove that a particular process is viable rather than considering the relative contributions of different processes (e.g. competition-Connell 1983, Quinn and Dunham 1983, Roughgarden 1983, Strong 1983). Although we argue that minimizing transport costs is unlikely to drive allometries over restricted mass ranges, it is easy to imagine that over broader size ranges, accumulating inefficiencies might disrupt the transport function (and its attendant benefits) of a vascular system. As the symmorphosis debate taught us, biological systems can tolerate mismatched efficiencies but not inadequate function (Dudley and Gans 1991, Jones 1998, Weibel et al. 1998). In general, a broader paradigm may allow the integration of proximate and ultimate mechanisms for allometry, to the benefit of both. We hope that such an approach will further invigorate and expand the consideration of the extent to which allometric effects constrain ecological processes (and vice versa) by expanding and integrating the mechanisms on which such hypotheses are based.

While the MTE has invigorated the study of mechanisms producing well known patterns of allometric scaling, it is our contention that this theory at best highlights potential physical constraints imposed on the allometry of metabolic rates. In our view, the MTE is in our view an oversimplified, insufficient description of the mechanisms underlying metabolic allometries, and cannot be considered a mechanistic explanation. While the MTE is a noble attempt to find general relationships in a very complex world, closer examination of the mechanisms proposed, as well as an accumulating body of literature surrounding the subject, suggests that our understanding of metabolic allometries will best be advanced by acknowledging that multiple causal mechanisms are likely to be involved.

\section{Unlinked Reference}

Kozlowski and Konarzewski, 2005

Acknowledgements - The ideas discussed here grew, in part, out of discussion groups at both Drexel Univ. and the Univ. of Pennsylvania. Thanks are due to all participants in both groups. We also thank Ed Dzialowski, Sue Kilham and Tony Steyermark, who read early versions of the manuscript and contributed ideas. We would also like to thank J. Brown who commented on an earlier version of the manuscript. F. H. was partially supported by a cooperative agreement from the United States Department of Agriculture's National Wildlife Research Center (NWRC) (03-7408-0479-CA) to the Univ. of Pennsylvania. We thank NWRC's Wildlife Disease Project: Surveillance, Monitoring, and Response for its continued support of this work.

\section{References}

Austad, S. N. 2005. Diverse aging rates in metazoans: targets for functional genomics. - Mech. Ageing Dev. 126: 4349.

Banavar, J. R. et al. 1999. Size and form in efficient transportation networks. - Nature 399: 130-132.

Banavar, J. R. et al. 2003. Allometric cascades. - Nature 421: 713-714.

Bejan, A. 2005. The constructal law of organization in nature: tree-shaped flows and body size. - J. Exp. Biol. 208: $1677-1686$.

Bennett, A. F. and Dawson, W. R. 1976. Metabolism. - In: Gans, C. and Dawson, W. R. (eds), Biology of the Reptilia. Vol. 5. Academic Press, pp. 127-224.

Bensusan, H. 1998. God doesn't always shave with Occam's razor - learning when and how to prune. - In: Lecture notes in computer science, Vol. 1398. Proc. 10th Eur. Conf. on Machine Learning. Springer, pp. 119-124.

Bentz, J. 1992. Intermediates and kinetics of membrane fusion. - Biophys. J. 63: 448-459.

Berne, R. M. et al. 2004. Physiology, 5th ed. - Mosby.

Beyer, E. C. and Spotila, J. R. 1994. Seasonal variation in metabolic rates and maintenance costs of the eastern fence lizard Sceloporus undulatus. - Comp. Biochem. Physiol. 109A: 1039-1047.

Biewener, A. A. 2005. Biomechanical consequences of scaling. - J. Exp. Biol. 208: 1665-1676.

Brown and West 2005. Please provide further information.

Brown, J. H. et al. 2004a. Toward a metabolic theory of ecology. - Ecology 85: 1771-1789.

Brown, J. H. et al. 2004b. Response to forum commentary on "Toward a metabolic theory of ecology". - Ecology 85: $1818-1821$

Brown, J. H. et al. 2005. Yes, West, Brown and Enquist's model of allometric scaling is both mathematically correct and biologically relevant. - Funct. Ecol. 19: 735-738.

Calder, W. A. III. 1984. Size, function and life history. - Harvard Univ. Press. 
Calder, W. A. III. 1987. Scaling energetics of homeothermic vertebrates: an operational allometry. - Annu. Rev. Physiol. 49: 107-120.

Cameron, J. N. 1989. The respiratory physiology of animals. - Oxford Univ. Press.

Chan, H. S. and Dill, K. A. 1998. Protein folding in the landscape perspective: Chevron plots and non-Arrhenius kinetics. - Proteins Struct. Funct. Genet. 30: 2-33.

Chaui-Berlinck, J. G. et al. 2005. Control of metabolic rate is a hidden variable in the allometric scaling of homeotherms. - J. Exp. Biol. 208: 1709-1716.

Clarke, A. 2004. Is there a universal temperature dependence of metabolism? - Funct. Ecol. 18: 252-256.

Clarke, A. and Fraser, K. P. P. 2004. Why does metabolism scale with temperature? - Funct. Ecol. 18: 243-251.

Connell, J. H. 1983. On the prevalence and relative importance of interspecific competition: evidence from field experiments. - Am. Nat. 122: 661-696.

Cottingham, K. L. and Zens, M. S. 2004. Metabolic rate opens a grand vista on ecology. - Ecology 85: 18051807.

Cyr, H. and Pace, M. I. 1993. Allometric theory-extrapolations from individuals to communities. - Ecology 74: 1234-1245.

Cyr, H. and Walker, S. C. 2004. An illusion of mechanistic understanding. - Ecology 85: 1802-1804.

Darveau, C. A. et al. 2002. Allometric cascade as a unifying principle of body mass effects on metabolism. - Nature 417: $166-170$.

Darveau, C. A. et al. 2003. Reply to Brown et al. - Nature 421: 714.

Dawson, T. H. 2001. Similitude in the cardiovascular system of mammals. - J. Exp. Biol. 204: 395-407.

Dawson, T. H. 2005. Modeling of vascular networks. - J. Exp. Biol. 208: 1687-1694.

Dawson, W. R. and Bartholomew, G. A. 1956. Relation of oxygen consumption to body weight, temperature and temperature acclimation in the lizards, Uta stansburiana and Sceloporus occidentalis. - Physiol. Zool. 29: 40-51.

Demetrius, L. 2003. Quantum statistics and allometric scaling of organisms. - Physica A 322: 477-490.

Dodds, P. S. et al. 2001. Re-examination of the '3/4-law' of metabolism. - J. Theor. Biol. 209: 9-27.

Dudley, R. and Gans, C. 1991. A critique of symmorphosis and optimality models in physiology. - Physiol. Zool. 64: 627-637.

Dunham, A. E. and Beaupre, S. J. 1998. Ecological experiments: scale, phenomenology, mechanism, and the illusion of generality. - In: Resitarits, W. and Bernardo, J. (eds), Experimental ecology: issues and perspectives. Oxford Univ. Press, pp. 27-40.

Dunham, A. E. et al. 1989. Interfaces between biophysical and physiological ecology and the population ecology of terrestrial vertebrate ectotherms. - Physiol. Zool. 62: $335-355$.

Else, P. L. and Hulbert, A. J. 1985. An allometric comparison of the mitochondria of mammalian and reptilian tissues: the implications for the evolution of endothermy. - J. Comp. Physiol. B 156: 3-11.

Fung, Y. C. 1997. Biomechanics: circulation, 2nd ed. - Springer.
Gates, D. M. 1980. Biophysical ecology. - Springer.

Gillooly, J. F. and Dodson, S. I. 2001. The relationship of neonate mass and incubation temperature to embryonic development time in a range of animal taxa. - J. Zool. Lond. 251: 369-375.

Gillooly, J. F. et al. 2001. Effects of size and temperature on metabolic rate. - Science 293: 2248-2251.

Gillooly, J. F. et al. 2002. Effects of size and temperature on developmental time. - Nature 417: 70-73.

Gillooly, J. F. et al. 2006. Response to Clarke and Fraser: effects of temperature on metabolic rate. - Funct. Ecol. 20: $400-404$.

Glazier, D. S. 2005. Beyond the '3/4-power law': variation in the intra- and interspecific scaling of metabolic rate in animals. - Biol. Rev. 80: 611-662.

Gordon, M. S. 1998. Evolution of optimal systems. - In: Weibel, E. R. et al. (eds), Principles of animal design: the optimization and symmorphosis debate. Cambridge Univ. Press, pp. 37-39.

Gotelli, N. 2001. A primer of eology, 3rd ed. - Sinauer.

Gould, S. J. and Lewontin, R. C. 1979. The spandrels of San Marco and the Panglossian paradigm: a critique of the adaptationist programme. - Proc. R. Soc. Lond. B 205: 581-598.

Gracey, A. Y. et al. 2004. Coping with cold: an integrative, multitissue analysis of the transcriptome of a poikilothermic vertebrate. - Proc. Natl Acad. Sci. USA 101: 1697016975.

Guderley, H. E. and St. Pierre, J. 1996. Phenotypic plasticity and evolutionary adaptations of mitochondria to temperature. - In: Johnston, I. A. and Bennett, A. F. (eds), Animals and temperature: phenotypic and evolutionary adaptation. Cambridge Univ. Press, pp. 127-152.

Guyton, A. C. and Hall, J. E. 2001. Textbook of medical physiology, 10th ed. - W. B. Saunders.

Harte, J. 2004. The value of null theories in ecology. - Ecology 85: 1792-1794.

Hill, R. W. et al. 2004. Animal physiology. - Sinauer.

Hochachka, P. W. et al. 2003. Allometric cascade: a model for resolving body mass effects on metabolism. - Comp. Biochem. Physiol. A 134: 675-691.

Huey, R. B. and Bennett, A. F. 1987. Phylogenetic studies of coadaptation: preferred temperatures versus optimal performance temperatures of lizards. - Evolution 41: 10981115.

Hulbert, A. J. and Else, P. L. 1981. Comparison of the "mammal machine" and the "reptile machine": energy use and thyroid activity. - Am. J. Phys. 241: R350-356.

Hulbert, A. J. and Else, P. L. 2000. Mechanisms underlying the cost of living in animals. - Annu. Rev. Physiol. 62: $207-235$.

Hulbert, A. J. and Else, P. L. 2005. Membranes and the setting of energy demand. - J. Exp. Biol. 208: 15931599.

Jeneson, J. A. L. et al. 2000. A metabolic control analysis of kinetic controls in ATP free energy metabolism in contracting skeletal muscle. - Am. J. Physiol. 279: C813-C832.

Jetz, W. et al. 2004. The scaling of animal space use. - Science 306: 266-268. 
Jones, J. H. 1998. Symmorphosis and the mammalian respiratory system: what is optimal design and does it exist? - In: Weibel, E. R. et al. (eds), Principles of animal design: the optimization and symmorphosis debate. Cambridge Univ. Press, pp. 241-248.

Kaspari, M. 2004. Using the metabolic theory of ecology to predict global patterns of abundance. - Ecology 85: 1800-1802.

Kingsolver, J. G. and Watt, W. B. 1984. Metabolic constraints and optimality models: thermoregulatory strategies in Colias butterflies. - Ecology 65: 1835-1839.

Kleiber., M. 1961. The fire of life, an introduction to animal energetics. - Wiley.

Koehl, M. A. R. and Wolcott, B. D. 2004. Can function at the organismal level explain ecological patterns? - Ecology 85: 1808-1810.

Kozlowski, J. 2000. Does body size optimization alter the allometries for production and life history traits? - In: Brown, J. H. and West, G. B. (eds), Scaling in biology. Oxford Univ. Press, pp. 237-252.

Kozlowski, J. and Weiner, J. 1997. Interspecific allometries are by-products of body size optimization. - Am. Nat. 149: 352-380.

Kozlowski and Konarzewski 2003. Please provide further information.

Kozlowski, J. and Konarzewski, M. 2004. Is West, Brown and Enquist's model of allometric scaling mathematically correct and biologically significant? - Funct. Ecol. 18: 283-289.

Kozlowski, J. and Konarzewski, M. 2005. West, Brown and Enquist's model of allometric scaling again: the same questions remain. - Funct. Ecol. 19: 739-743.

Lesser, M. P. and Kruse, V. A. 2004. Seasonal temperature compensation in the horse mussel, Modiolus modiolus: metabolic enzymes, oxidative stress and heat shock proteins. - Comp. Biochem. Physiol. A 137: A495A504.

Li, J. K-J. 2000. Scaling and invariants in cardiovascular biology. - In: Brown, J. H. and West, G. B. (eds), Scaling in biology. Oxford Univ. Press, pp. 113-128.

Lindeman, R. 1942. The trophic-dynamic aspect of ecology. - Ecology 23: 399-418.

Makarieva, A. M. et al. 2005. Energetics of the smallest: do bacteria breathe at the same rate as whales? - Proc. R. Soc. B 272: 2219-2224.

Marquet, P. A. et al. 2004. Metabolic ecology: linking individuals to ecosystems. - Ecology 85: 1794-1796.

Martin, R. D. et al. 2005. Problems of allometric scaling analysis: examples from mammalian reproductive biology. - J. Exp. Biol. 208: 1731-1747.

McMahon, T. A. 1973. Size and shape in biology. - Science 179: $1201-1204$.

McMahon, T. A. 1975 . Using body size to understand the structural design of animals: quadrupedal locomotoion. - J. Appl. Physiol. 39: 619-627.

Moyes, C. D. and LeMoine, C. M. R. 2005. Control of muscle bioenergetic gene expression: implications for allometric scaling relationships of glycolytic and oxidative enzymes. - J. Exp. Biol. 208: 1601-1610.
Muller-Landau, H. et al. 2006. Testing metabolic ecology theory for allometric scaling of tree size, growth and mortality in tropical forests. - Ecol. Lett. 9: 575-588.

Nagy, K. A. 2005. Field metabolic rate and body size. - J. Exp. Biol. 208: 1621-1625.

Niewiarowski, P. H. and Dunham, A. E. 1994. The evolution of reproductive effort in squamate reptiles-costs, tradeoffs, and assumptions reconsidered. - Evolution 48: 137-145.

O'Connor, M. P. 1999. Physiological and ecological implications of a simple model of heating and cooling in reptiles. - J. Thermal Biol. 24: 113-136.

O'Connor, M. P. and Dodson, P. 1999. Biophysical constraints on the thermal ecology of dinosaurs. - Paleobiology 25: 341-368.

O'Connor, M. P. et al. 2006. Linking physiological effects on activity and resource use to population level phenomena. - Integrative Comp. Biol. 46: 1093-1109.

Patronek, G. J. et al. 1997. Comparative longevity of pet dogs and humans: implications for gerontology research. - J. Gerontol. A 52: B171-B178.

Peters, R. H. 1983. The ecological implications of body size. - Cambridge Univ. Press.

Peters, R. H. 1991. A critique for ecology. - Cambridge Univ. Press.

Pierce, G. J. and Ollason, J. G. 1987. Eight reasons why optimal foraging theory is a complete waste of time. - Oikos 49: 111-118.

Porter, W. P. et al. 2002. Physiology on a landscape scale: plant-animal interactions. - Integrative Comp. Biol. 42: 431-453.

Pough, F. H. 1980. The advantages of ectothermy for tetrapods. - Am. Nat. 115: 92-112.

Pyke, G. H. 1984. Optimal foraging theory. - Annu. Rev. Ecol. Syst. 15: 523-575.

Quinn, J. F. and Dunham, A. E. 1983. On hypothesis testing in ecology and evolution. - Am. Nat. 122: 602-617.

Reich et al., 2004. Please provide further information.

Reich, P. B. et al. 2006. Universal scaling of respiratory metabolism, size and nitrogen in plants. - Nature 439: 457-461.

Rolfe, D. F. S. and Brown, G. C. 1997. Cellular energy utilization and molecular origin of standard metabolic rate in mammals. - Physiol. Rev. 77: 731-758.

Roughgarden, J. 1983. Competition and theory in community ecology. - Am. Nat. 122: 583-601.

Savage, V. M. et al. 2004. The predominance of quarter power scaling in biology. - Funct. Ecol. 18: 257-282.

Schmidt-Nielsen, K. 1984. Why is animal size so important?. - Cambridge Univ. Press.

Speakman, J. R. 2005. Body size, energy metabolism and lifespan. - J. Exp. Biol. 208: 1717-1730.

Speakman, J. R. et al. 2003. Age-related changes in the metabolism and body composition of three dog breeds and their relationship to life expectancy. - Aging Cell 2: $265-275$.

Speakman, J. R. et al. 2004. Uncoupled and surviving: individual mice with high metabolism have greater mitochondrial uncoupling and live longer. - Aging Cell 3: 87-95. 
Strong, D. R. 1983. Natural variability and the manifold mechanisms of ecological communities. - Am. Nat. 122: 636-660.

Suarez, R. K. and Darveau, C. A. 2005. Multi-level regulation and metabolic scaling. - J. Exp. Biol. 208: 1627-1634.

Taylor, C. R. and Wiebel, E. R. 1981. Design of the mammalian respiratory system. - Respiration Physiol. 44: $1-10$.

Taylor, C. R. et al. 1981. Design of the mammalian respiratory system. III. Scaling maximum aerobic capacity to body mass: wild and domestic mammals. - Respiration Physiol. 44: 25-37.

Taylor, E. W. 1998. Gills of water breathers: structures with multiple functions. - In: Weibel, E. R. et al. (eds), Principles of animal design: the optimization and symmorphosis debate. Cambridge Univ. Press, pp. 186-194.

Tilman, D. et al. 2004. Does metabolic theory apply to community ecology? It's a matter of scale. - Ecology 85: 1797-1799.

Tu, M. and Tatar, M. 2003. Juvenile diet restriction and the aging and reproduction of adult Drosophila melanogaster. - Aging Cell 2: 327-333.

Van Voorhies, W. A. 2004. Live fast, live long? A commentary on a recent paper by Speakman et al. - Aging Cell 3: $327-330$.

Vogel, S. 1994. Life in moving fluids. - Princeton Univ. Press.

Vogel, S. 2000. Life's devices: the physical world of animals and plants. - Princeton Univ. Press.

Webb, G. I. 1996. Further experimental evidence against the utility of Occam's razor. - J. Artificial Intelligence Res. 4: 397-417.

Weibel, E. R. and Hoppeler, H. 2005. Exercise-induced maximal metabolic rate scales with muscle aerobic capacity. - J. Exp. Biol. 208: 1635-1644.
Weibel, E. R. et al. 1998. Principles of animal design: the optimization and symmorphosis debate. - Cambridge Univ. Press.

West and Brown, 2003. Please provide further information.

West, G. B. and Brown, J. H. 2005. The origin of allometric scaling laws in biology from genomes to ecosystems: towards a quantitative unifying theory of biological structure and organization. - J. Exp. Biol. 208: 15751592.

West, G. B. et al. 1997. A general model for the origin of allometric scaling laws in biology. - Science 276: $122-$ 126.

West, G. B. et al. 1999. The fourth dimension of life: fractal geometry and allometric scaling of organisms. - Science 284: $1677-1679$.

West, G. B. et al. 2003. Why does metabolic rate scale with body size? - Nature 421: 713.

West, G. B. et al. 2004. Growth models based on first principles or phenomenology? - Funct. Ecol. 18: 188196.

White, C. R. and Seymour, R. S. 2003. Mammalian basal metabolic rate is proportional to body mass ${ }^{2 / 3}$. - Proc. Natl Acad. Sci. USA 100: 4046-4049.

White, C. R. and Seymour, R. S. 2005. Allometric scaling of mammalian metabolism. - J. Exp. Biol. 208: 16111619.

Whiteley, N. and Faulkner, L. S. 2005. Temperature influences whole-animal rates of metabolism but not protein synthesis in a temperate intertidal isopod. - Physiol. Biochem. Zool. 78: 227-238.

Zakhartsev, M. et al. 2004. Effects of temperature acclimation on lactate dehydrogenase of cod Gadus morhua.: genetic, kinetic and thermodynamic aspects. - J. Exp. Biol. 207: 95-112. 